\title{
INTERPOLATION OF $l^{q}$ SEQUENCES BY $H^{p}$ FUNCTIONS
}

\author{
B. A. TAYLOR AND D. L. WILLIAMS
}

Abstract. It is pointed out that the method used by $L$. Carleson to study interpolation by bounded analytic functions applies to the study of the analogous problem for $H^{p}$ functions. In particular, there exist sequences of points in the unit disc which are not uniformly separated, but which are such that every $l^{q}$ sequence can be interpolated along this sequence by an $H^{p}$ function $(1 \leqq p \leqq q \leqq$ $+\infty)$.

Let $\left(H^{p}, l^{q}\right)$ denote the set of sequences $\left\{z_{n}\right\}_{n=1}^{\infty}$ in the open unit disc such that

$$
\left\{\left\{f\left(z_{n}\right)\right\}: f \in H^{p}\right\} \supset l^{q} .
$$

It was proved by L. Carleson [1] and also by H. S. Shapiro and A. L. Shields [7] that $\left\{z_{n}\right\} \in\left(H^{\infty}, l^{\infty}\right)$ if and only if $\left\{z_{n}\right\}$ is uniformly separated; that is,

$$
\prod_{k ; k \neq n}\left|\frac{z_{n}-z_{k}}{1-\bar{z}_{k} z_{n}}\right| \geqq \delta>0, \quad n=1,2, \cdots .
$$

Since $H^{\infty} \subset H^{p}$ for all $p<\infty,\left(H^{p}, l^{\infty}\right)$ contains the uniformly separated sequences. A. K. Snyder [8] has proved the existence of sequences $\left\{z_{n}\right\} \in$ $\left(H^{2}, l^{\infty}\right)$ which are not uniformly separated, and P. L. Duren and H. S. Shapiro [4] have given for $0<p<\infty$ an explicit construction of sequences $\left\{z_{n}\right\}$ which belong to $\left(H^{p}, l^{\infty}\right)$ but which are not uniformly separated.

We show here that the method used by Carleson [1], as modified by Hörmander [5] and extended by Duren [2], also applies directly to the problem of characterizing $\left(H^{p}, l^{q}\right)$ when $1 \leqq p, q \leqq \infty$. In particular, this method allows one to construct easily sequences in $\left(H^{p}, l^{\infty}\right)$ which are not uniformly separated.

Carleson's method is to convert the interpolation problem to one of estimating a measure associated with the sequence $\left\{z_{n}\right\}$. The equivalence is given by the following theorem whose proof is a well-known consequence of the $L^{p}-L^{p^{\prime}}$ duality. See, for example [3, Chapter 8].

Received by the editors January 8, 1971 and, in revised form, October 4, 1971. AMS 1970 subject classifications. Primary 30A78, 30A80.

Key words and phrases. Interpolation, $H^{p}$ spaces, analytic functions, subharmonic functions.

(c) American Mathematical Society 1972 
TheOrem 1. Assume $1 \leqq p, q \leqq \infty$. The sequence $\left\{z_{n}\right\} \in\left(H^{p}, l^{q}\right)$ if and only if

(i) $\sum_{n}\left(1-\left|z_{n}\right|\right)<\infty$,

(ii) for some constant $M>0$,

$$
\left(\sum_{n}\left|\frac{F\left(z_{n}\right)}{B^{\prime}\left(z_{n}\right)}\right|^{q^{\prime}}\right)^{1 / q^{\prime}} \leqq M\|F\|_{p^{\prime}}, \quad F \in H^{p^{\prime}},
$$

where $p^{\prime}, q^{\prime}$ are the conjugate indices of $p, q$ respectively, and $B$ is the Blaschke product with zeros $\left\{z_{n}\right\}$.

Let $\mu=\sum_{n}\left|B^{\prime}\left(z_{n}\right)\right|^{-q} \delta_{z_{n}}$, where $\delta_{z_{n}}$ is the unit point mass at $z_{n}$. Then the estimate (ii) takes the form (note that we have now replaced $p^{\prime}$ by $p$ and $q^{\prime}$ by $q$ )

$$
\left(\int|F(z)|^{q} d \mu(z)\right)^{1 / q} \leqq M\left(\frac{1}{2 \pi} \int_{-\pi}^{\pi}\left|F\left(e^{i \theta}\right)\right|^{p} d \theta\right)^{1 / p}, \quad F \in H^{p} .
$$

Carleson [1] gave the necessary and sufficient condition on the measure $\mu$ in order that (1) hold in the cases $0<p=q<\infty$. Duren [2] has used a method of Hörmander [5] to extend Carleson's theorem to the cases $0<p \leqq q<\infty$. We shall show how the same method can be modified to obtain information in the cases $0<q<p<\infty$.

For each $z=r e^{i \theta}$ in the unit disc, let

$$
I_{z}=\left\{e^{i t}:|\theta-t|<\frac{1}{2}(1-r)\right\},
$$

and for an open subset $U \subset\{|z|=1\}$ let

$$
S(U)=\left\{z: I_{z} \subset U\right\} .
$$

Let $m$ denote Lebesgue measure on $\{|z|=1\}$.

TheOREM 2. Assume $0<q<p<\infty$ and let $\mu$ be any positive Borel measure in the open unit disc. If (1) holds, then there is a constant $C$ such that for every open subset $U \subset\{|z|=1\}$,

$$
\mu(S(U)) \leqq C[m(U)]^{q / p} .
$$

In order for (1) to hold, it is sufficient that (2) hold with q/p replaced by $q / p+\varepsilon$, for some $\varepsilon>0$.

REMARKS. When $0<p \leqq q<\infty$, the estimate (2) is implied by the apparently weaker estimate

$$
\mu(S(I)) \leqq C[m(I)]^{a / p}
$$

for all open arcs $I \subset\{|z|=1\}$. For, if $U=\bigcup_{n} I_{n}$ where the $I_{n}$ are disjoint 
open arcs, then

$$
\begin{aligned}
{[\mu(S(U))]^{p / q} } & =\left[\sum_{n} \mu\left(S\left(I_{n}\right)\right)\right]^{p / q} \\
& \leqq \sum_{n}\left[\mu\left(S\left(I_{n}\right)\right)\right]^{p / q} \leqq C \sum_{n} m\left(I_{n}\right)=C m(U),
\end{aligned}
$$

where the first inequality follows from $p / q \leqq 1$.

When $0<p \leqq q<\infty$, the theorems of Carleson and Duren show that (3) is necessary and sufficient for (1) to hold. This is not the case when $0<$ $q<p<\infty$. For example, let $d \mu=(1-x)^{\alpha / p-1} d x$ (supported on $(0,1)$ in $\{|z|<1\})$, and let $F(z)=(1-r z)^{-1 / p}$. Then both (2) and (3) are satisfied, but

while

$$
\begin{aligned}
\left(\int_{-\pi}^{\pi}|F|^{\alpha} d \mu\right)^{1 / q} & =\left(\int_{0}^{1}(1-r x)^{-q / p}(1-x)^{q / p-1} d x\right)^{1 / q} \\
& \geqq(-\log (1-r))^{1 / q}
\end{aligned}
$$

$$
\left(\frac{1}{2 \pi} \int_{-\pi}^{\pi}\left|F\left(e^{i \theta}\right)\right|^{p} d \theta\right)^{1 / p}=O\left((-\log (1-r))^{1 / p}\right), \quad r \rightarrow 1^{-} .
$$

Therefore (1) fails for this $\mu$.

Proof of Theorem 2. The proof follows closely that of Hörmander [5] and Duren [2]. and so we shall only outline it.

By factoring out Blaschke products and taking appropriate powers one shows that (1) is equivalent to

$$
\left(\int|F|^{2} d \mu\right)^{1 / 2} \leqq M^{q / 2}\|F\|_{\beta}, \quad F \in H^{\beta},
$$

where $\beta=2 p / q>2$. The Riesz conjugacy theorem [3, Chapter 4] implies that (4) also holds (with a different constant) for harmonic functions which are extensions to $\{|z|<1\}$ of $L^{\beta}$ functions on $\{|z|=1\}$. The necessity of (2) then follows by applying this estimate to the Poisson integral of the characteristic function of $U$.

To prove the sufficiency part of the theorem, one establishes a weak type estimate and then applies an interpolation theorem to obtain the strong type estimate (4).

Our hypothesis is that

$$
\mu(S(U)) \leqq C[m(U)]^{1 / \alpha}
$$

where $1 / \alpha=q \mid p+\varepsilon<1$ for some $\varepsilon>0$. Let $f$ be an $L^{\alpha}$ function on $\{|z|=1\}$, and let

$$
u^{*}(z)=\sup \frac{1}{m(I)} \int_{I}\left|f\left(e^{i t}\right)\right| d t
$$


where the supremum is taken over all arcs $I \subset\{|z|=1\}$ with $I_{z} \subset I$. Then there is a constant $C^{\prime}$ such that

$$
\mu\left\{u^{*}(z)>h\right\} \leqq C^{\prime} h^{-1}\|f\|_{\alpha}, \quad f \in L^{\alpha} .
$$

The argument used to prove the weak type $(\alpha, 1)$ estimate $(6)$ follows generally the arguments in [2] and [5]; therefore, we shall not give the details. Note also the strong type $(\infty, \infty)$ estimate $\sup _{|z|<1} u^{*}(z) \leqq\|f\|_{\infty}$.

We thus have a sublinear operator $f \rightarrow u^{*}$ which is of weak type $(\alpha, 1)$ and strong type $(\infty, \infty)$. Now the Marcinkiewicz interpolation theorem does not apply here; however, the weak type interpolation theorem for Lorentz spaces [6, p. 264] implies that, for each $\eta>0$ and each $\gamma>1$, there exists a constant $C_{\eta, \gamma}$ such that

$$
\left(\int\left[u^{*}(z)\right]^{\gamma} d \mu(z)\right)^{1 / \gamma} \leqq C_{\eta, \gamma}\|f\|_{\alpha \gamma+\eta}, \quad f \in L^{\alpha \gamma+\eta} .
$$

Taking $\gamma=2$ and choosing $\eta$ so that $\alpha \gamma+\eta=\beta$, we have

$$
\left(\int\left[u^{*}(z)\right]^{2} d \mu\right)^{1 / 2} \leqq C_{\beta}\|f\|_{\beta}, \quad f \in L^{\beta} .
$$

For $F \in H^{\beta},|F(z)| \leqq u(z) \leqq$ const $u^{*}(z)$ where $u$ is the harmonic extension of $|F|$ from $\{|z|=1\}$ into $\{|z|<1\}$. Thus, (4) follows, and the theorem is proved.

We shall now construct an example of a sequence in $\left(H^{p}, l^{\infty}\right)$ which is not uniformly separated. Here $1 \leqq p<\infty$. Let $\left\{z_{n}\right\}$ be a sequence in $\{|z|<1\}$ such that $0<\operatorname{Re} z_{n} \nearrow 1$ and $\operatorname{Im} z_{n} \searrow 0$. In addition assume that $\left\{z_{n}\right\}$ is uniformly separated and $\left|z_{n}-\bar{z}_{n}\right| \sim\left(1-\left|z_{n}\right|^{2}\right)^{2-1 / p^{\prime}-\varepsilon}$ where $0<$ $1 / p^{\prime}+\varepsilon<1$. Clearly $\left\{z_{n}, \bar{z}_{n}\right\}$ is not uniformly separated. We shall show $\left\{z_{n}, \bar{z}_{n}\right\} \in\left(H^{p}, l^{\infty}\right)$. Let $B$ be the Blaschke product associated with $\left\{z_{n}, \bar{z}_{n}\right\}$. From the uniform separation of $\left\{z_{n}\right\}$, it follows easily that $\left(1-\left|z_{n}\right|^{2}\right) \leqq$ $\delta^{-1}$ const $\left(r_{n+1}-r_{n}\right)$, where $\left|z_{n}\right|=r_{n}$. We will prove that, for some constant $K$,

(8) $\sum_{r_{n} \geqq r}\left[\left|B^{\prime}\left(z_{n}\right)\right|^{-1}+\left|B^{\prime}\left(\bar{z}_{n}\right)\right|^{-1}\right]=2 \sum_{r_{n} \geqq r}\left|B^{\prime}\left(z_{n}\right)\right|^{-1} \leqq K(1-r)^{1 / p^{\prime}+\varepsilon}$.

This inequality shows that the measure

$$
\mu=\sum_{n}\left(\left|B^{\prime}\left(z_{n}\right)\right|^{-1} \delta_{z_{n}}+\left|B^{\prime}\left(\bar{z}_{n}\right)\right|^{-1} \delta_{\bar{z}_{n}}\right)
$$

satisfies (2) with exponent $1 / p^{\prime}+\varepsilon$, because for any open subset $U$ of $\{|z|=1\}$, the left-hand side is the $\mu$ measure of the entire annulus $r \leqq|z| \leqq 1$ and on the right-hand side, $1-r$ is the length of the largest of the arcs constituting $U$. Therefore, Theorem 2 implies that the hypothesis (ii) of Theorem 1 holds and, consequently, that $\left\{z_{n}, \bar{z}_{n}\right\} \in\left(H^{p}, l^{\infty}\right)$. 
The inequality (8) holds because

$$
\begin{aligned}
& 2 \sum_{r_{n} \geqq r}\left|B^{\prime}\left(z_{n}\right)\right|^{-1} \\
& \quad=2 \sum_{r_{n} \geqq r}\left(1-\left|z_{n}\right|^{2}\right)\left|\frac{1-\left|z_{n}\right|^{2}}{z_{n}-\bar{z}_{n}}\right| \prod_{k ; k \neq n}\left|\frac{1-\bar{z}_{k} z_{n}}{z_{n}-z_{k}}\right| \prod_{k ; k \neq n}\left|\frac{1-\bar{z}_{k} \bar{z}_{n}}{\bar{z}_{n}-z_{k}}\right| \\
& \quad \leqq 2 \delta^{-2} \sum_{r_{n} \geqq r}\left(1-\left|z_{n}\right|^{2}\right)^{2}\left|z_{n}-\bar{z}_{n}\right|^{-1} \\
& \quad \leqq \delta^{-3} \text { const } \sum_{r_{n} \geqq r}\left(1-r_{n}^{2}\right)^{1 / p^{\prime}+\varepsilon-1}\left(r_{n+1}-r_{n}\right) \\
& \quad \leqq \delta^{-3} \text { const } \int_{r}^{1}\left(1-t^{2}\right)^{1 / p^{\prime}+\varepsilon-1} d t \leqq K(1-r)^{1 / p^{\prime}+\varepsilon} .
\end{aligned}
$$

We also remark that it is not hard to show that one can always add an infinite number of points to any uniformly separated sequence so that the resulting sequence is not uniformly separated, but still belongs to $\left(H^{p}, l^{q}\right)$ $(1<p<q \leqq+\infty)$.

One may also use the techniques of this paper to study weighted interpolation. Let $W\left(H^{p}, l^{q}\right)$ denote the sequences $\left\{z_{n}\right\}$ such that

$$
\left\{\left\{f\left(z_{n}\right)\left(1-\left|z_{n}\right|\right)^{1 / a}\right\}: f \in H^{p}\right\} \supset l^{q} .
$$

H. S. Shapiro and A. L. Shields [7] have shown that for $1 \leqq p \leqq \infty$, $\left\{z_{n}\right\} \in W\left(H^{p}, l^{p}\right)$ if and only if $\left\{z_{n}\right\}$ is uniformly separated (in fact, equality holds in (9) when $\left\{z_{n}\right\}$ is uniformly separated). A theorem similar to Theorem 1 holds for this type of weighted interpolation. In fact, one only needs to replace the estimate (ii) by

$$
\left(\sum_{n}\left|\frac{F\left(z_{n}\right)}{B^{\prime}\left(z_{n}\right)}\right|^{q^{\prime}}\left(1-\left|z_{n}\right|\right)^{1-q^{\prime}}\right)^{1 / q^{\prime}} \leqq M\|F\|_{p^{\prime}}
$$

Consequently, we may apply Theorem 2 where the relevant measure is

$$
\mu=\sum_{n=1}^{\infty}\left(1-\left|z_{n}\right|\right)^{1-q^{\prime}}\left|B^{\prime}\left(z_{n}\right)\right|^{-q^{\prime}} \delta_{z_{n}}
$$

For $1<p<q \leqq \infty$ a slight modification of the above example yields a $W\left(H^{p}, l^{q}\right)$ sequence which is not uniformly separated. Simply alter the above example by choosing $\varepsilon>0$ so that $q^{\prime} \mid p^{\prime}+\varepsilon<1$ and making $\left|z_{n}-\bar{z}_{n}\right| \sim$ $\left(1-\left|z_{n}\right|^{2}\right)^{1+\left(1 / q^{\prime}\right)\left(1-q^{\prime} / p^{\prime}-\varepsilon\right)}$.

\section{REFERENCES}

1. L. Carleson, An interpolation problem for bounded analytic functions, Amer. J. Math. 80 (1958), 921-930. MR 22 \#8129.

2. P. L. Duren, Extension of a theorem of Carleson, Bull. Amer. Math. Soc. 75 (1969), 143-146. MR 39 \#2989. 
3. P. L. Duren, Theory of $H^{p}$ spaces, Pure and Appl. Math., vol. 38, Academic Press, New York, 1970. MR 42 \#3552.

4. P. L. Duren and H. S. Shapiro, Interpolation in $H^{p}$ spaces, Proc. Amer. Math. Soc. 31 (1972), 162-164.

5. L. Hörmander, $L^{p}$ estimates for (pluri-) subharmonic functions, Math. Scand. 20 (1967), 65-78. MR 38 \#2323.

6. R. A. Hunt, On $L(p, q)$ spaces, Enseignement Math. (2) 12 (1966), 249-276. MR 36 \#6921.

7. H. S. Shapiro and A. L. Shields, On some interpolation problems for analytic functions, Amer. J. Math. 83 (1961), 513-532. MR 24 \#A3280.

8. A. K. Snyder, Sequence spaces and interpolation problems for analytic functions, Studia Math. (to appear).

Department of Mathematics, University of Michigan, Ann Arbor, Michigan 48104

Department of Mathematics, Syracuse University, Syracuse, New York 13210 